\title{
Nanoscale
}

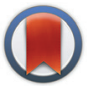

CrossMark \& click for updates

Cite this: Nanoscale, 2015, 7, 11263

Received 10th May 2015, Accepted 29th May 2015

DOI: $10.1039 /$ c5nr03058g

www.rsc.org/nanoscale

\section{Two-colour fluorescent imaging in organisms using self-assembled nano-systems of upconverting nanoparticles and molecular switches $\uparrow$}

\author{
Tuoqi Wu, ${ }^{a}$ Bob Johnsen, ${ }^{\mathrm{b}}$ Zhaozhao Qin, ${ }^{\mathrm{b}}$ Masakazu Morimoto, ${ }^{\mathrm{c}}$ David Baillie, ${ }^{\text {tb }}$ \\ Masahiro Irie ${ }^{\star c}$ and Neil R. Branda*a
}

The water-insolubility of a potentially versatile photoresponsive 'turn-on' fluorescence probe was overcome by incorporating it into a nano-assembly containing an upconverting nanoparticle wrapped in an amphiphilic polymer. The appeal of the nanosystem is not only in the ability to turn "on" and "off" the fluorescence from the organic chromophore using UV and visible light, it is in the fact that the nanoparticle acts as a static probe because it emits red and green light when excited by near infrared light, which is not effected by UV and visible light. This dual-functioning emission behaviour was demonstrated in live organisms.

\section{Introduction}

Although the use of conventional fluorescent probes ${ }^{1}$ for bioimaging applications has proven experimentally and clinically beneficial, the use of 'turn-on' fluorescent probes ${ }^{2}$ has its own merits. These probes are engineered to emit read-out signals only after they are activated by an external stimulus. ${ }^{2}$ When the external stimulus involves a chemical reaction or interaction, the appearance of emission is a convenient way to validate site-specific binding or detect biological events in cells or living organisms. On the other hand, photoresponsive probes that are turned 'on' and 'off' using two colours of stimulating light when and where the user desires adds the component of time to imaging methods. This benefit offers the ability to separate the spatially indistinguishable fluorophores temporally, and allows the reconstruction of images with subdiffraction

\footnotetext{
${ }^{a} 4 D$ LABS, Department of Chemistry, Simon Fraser University, 8888 University Drive, Burnaby, BC, Canada V5A 1S6. E-mail: nbranda@sfu.ca; Fax: +1 778 782-3765; Tel: $+1778782-8051$

${ }^{b}$ Department of Molecular Biology and Biochemistry, Simon Fraser University, 8888 University Drive, Burnaby, BC, Canada V5A 1S6

${ }^{c}$ Department of Chemistry and Research Center for Smart Molecules, Rikkyo University, 3-34-1 Nishi-Ikebukuro, Toshima-ku, Tokyo 171-8501, Japan $\dagger$ Electronic supplementary information (ESI) available: Detailed descriptions of experimental methods, synthetic procedures, characterization of new compounds and additional absorption spectra. See DOI: 10.1039/c5nr03058g
}

resolution to improve the quality of images. ${ }^{3}$ These features explain why 'turn-on' fluorescent probes are frequently used as tools for cell imaging where they can detect chemical species ${ }^{4}$ and imaging proteins in cells, ${ }^{5}$ while photoactive 'turn-on' fluorescent probes can be used for super-resolution imaging. ${ }^{3}$

A recently reported example of a probe that changes from a dark, non-emissive state to a bright, emissive state was based on the reversible photochemical reaction of the disulfone 10 shown in Scheme $1 .^{6}$ This appealing system has an impressive emission contrast ratio between its two states and is based on the well known ring-closing and ring-opening reactions of diarylethenes using two different colours of light. ${ }^{7}$ In the case of this diarylethene, its non-emissive ring-open isomer $\mathbf{1 0}$ is quantitatively converted to its highly emissive ring-closed isomer 1c using UV light $(\mathbf{1 o} \rightarrow \mathbf{1 c})$. The broad emission of $\mathbf{1 c}$ is between $500 \mathrm{~nm}$ and $650 \mathrm{~nm}$ when using light of $488 \mathrm{~nm}$ as the excitation source. The emission can be turned off using visible light, which triggers the ring-opening reaction $(\mathbf{1 c} \rightarrow$ 10) and regenerates the original non-emissive compound.

We have demonstrated how multi-component, watersoluble nano-systems are self-assembled when an amphiphilic polymer wraps itself around inorganic upconverting nanoparticles (UCNPs). ${ }^{8}$ The amphiphilic nature of the polymer ensures the entire nano-system has good compatibility with aqueous environments while providing a lipophilic layer close to the surface of the nanoparticles. It is within this lipophilic

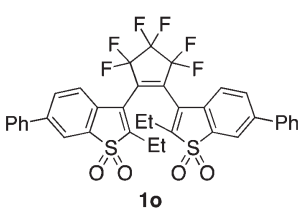

does not absorb $488 \mathrm{~nm}$ light non-fluorescent $\left(\lambda_{\text {ex }}=488 \mathrm{~nm}\right)$
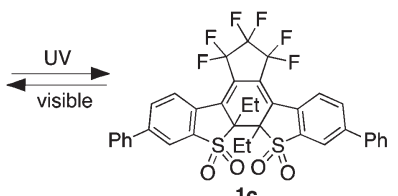

$1 c$

absorbs $488 \mathrm{~nm}$ light fluorescent $\left(\lambda_{\mathrm{ex}}=488 \mathrm{~nm}\right)$
Scheme 1 Photoinduced ring-closing and ring-opening reactions of the diarylethene (1o and $1 \mathrm{c}$ ). 
layer that hydrophobic compounds such as $\mathbf{1 0}$ are entrapped if they are present during the self-assembly process.

In this article, we describe the 'turn-on' properties of a selfassembled nano-system containing compound 10, which becomes reversibly emissive when exposed to UV and visible light. The appeal of the system is that the nanoparticles display their own emission when they absorb several photons of near-infrared (NIR) light and convert them into red and green ones. ${ }^{9}$ The resulting system is multi-modal (it has two fluorescent probes). The emission from the UCNPs (stimulated by NIR light) is always 'on' and can always be used as a readout signal even when the signal from the 'turn-on' probe is 'off'. Using UV light to trigger the ring-closing reaction of $\mathbf{1 0}$ and consequently turning the emission from the organic chromophore 'on' provides a greater level of reliability and a reduction in false positive signals when tracking biochemical events. ${ }^{10}$ We demonstrate the use of our nano-system imaging agent in live Caenorhabditis elegans nematodes.

\section{Results and discussion}

The self-assembled nano-system 1o-NP is shown in Fig. 1 and was synthesized using the reported one-pot protocol ${ }^{8 d}$ by treat-

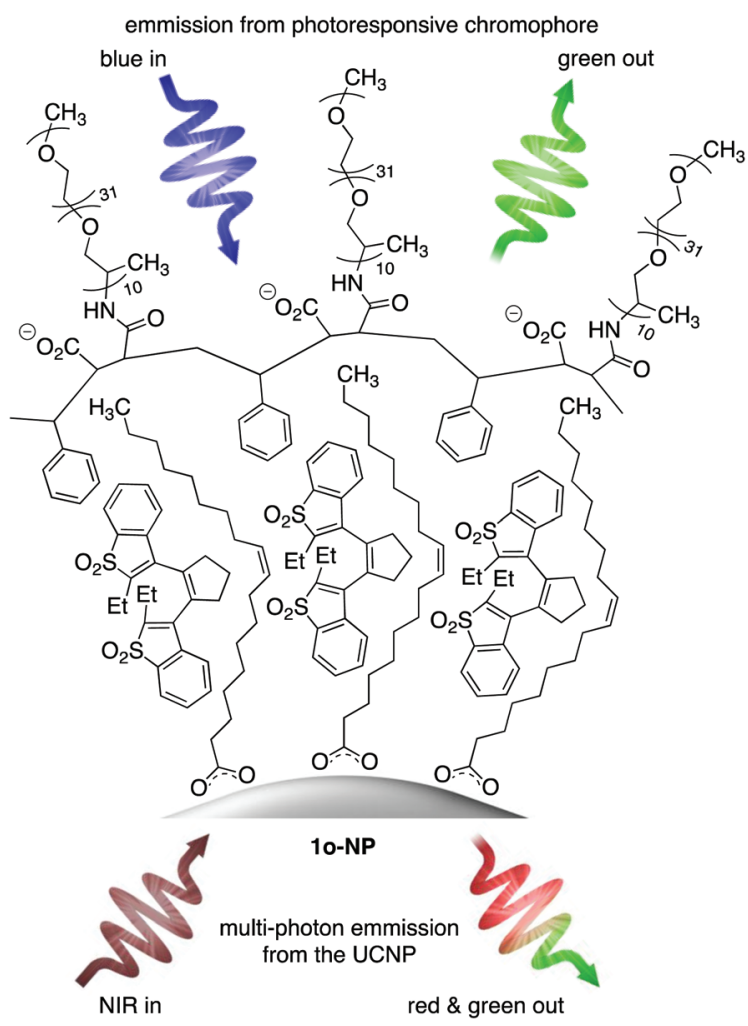

Fig. 1 The self-assembled nano-system (10-NP) containing the amphiphilic polymer wrapped around the lanthanide-doped upconverting nanoparticles with encapsulated diarylethene chromophores. The green emission from the organic chromophore is turned 'on' using UV light to ring-close the diarylethene. The multiphoton emission from the UCNPs is always on when irradiated with NIR light. (a)
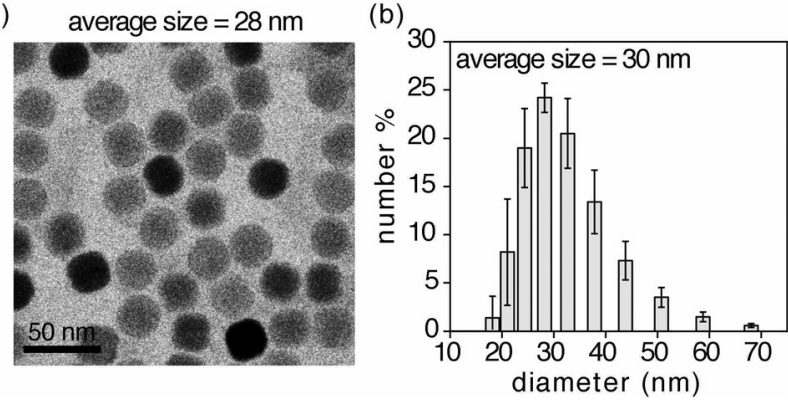

Fig. 2 (a) TEM image of the nano-assemblies (10-NP). (b) DLS measurements of the size distribution of the nano-assembles. The average sizes calculated using each technique are provided. For the DLS measurements, the original aqueous dispersion $\left(0.4 \mathrm{mg} \mathrm{mL}^{-1}\right.$ as determined by TGA; $0.8 \times 10^{-6} \mathrm{M} 10$ ) was diluted 100 times with water and this diluted sample was employed for the measurements. The sample was incubated at $25^{\circ} \mathrm{C}$ for $2 \mathrm{~min}$ before the DLS measurements were acquired.

ing a $\mathrm{CHCl}_{3}$ dispersion of oleate-coated lanthanide-doped (2 $\mathrm{mol} \% \mathrm{Er}^{3+}$ and $20 \% \mathrm{Yb}^{3+}$ ) upconverting nanoparticles $\left(\mathrm{NaYF}_{4}: \mathrm{ErYb}\right)$ and poly(styrene-co-maleic anhydride) with poly (propylene glycol)bis(2-aminopropyl ether) to ring-open the anhydride groups. Introducing the hydrophobic chromophore (10) and replacing the organic solvent with basic water promotes the self-assembly process and produces the nano-system 10-NP, which were isolated by passing the aqueous sample through syringe filters.

Transmission electron microscopy (TEM) images of 10-NP (Fig. 2a) show an average diameter of $28 \mathrm{~nm}$ for the nanosystems (Fig. S1 $\dagger$ ). The average size is measured to be slightly larger $(30 \mathrm{~nm})$ by dynamic light scattering (DLS) because this technique measures the average hydrodynamic radius, which includes the polymer shell (Fig. 2b). This organic shell cannot be directly observed by TEM due to its lack of contrast on the TEM grid. The shell thickness of $2 \mathrm{~nm}$ is substantially smaller than what it would be if all the polyethylene glycol chains were fully extended $(11 \mathrm{~nm})$ suggesting the polymer shell exists in a compressed form as has been previously observed. ${ }^{8 a, b}$

As is typically done for photochromic compounds, the ringclosing (10-NP $\rightarrow$ 1c-NP) and ring-opening (1c-NP $\rightarrow$ 10-NP) reactions of the chromophores entrapped within the nanoassemblies are best monitored using UV-vis absorption spectroscopy. When an aqueous dispersion of 10-NP is exposed to $365 \mathrm{~nm}$ light, $\S$ the characteristic bands in the visible region of the spectrum (centered at $460 \mathrm{~nm}$ ) corresponding to the ringclosed isomer 1c appear (Fig. 3a). These bands overlap with those for the ring-closed isomer of the free chromophore in $\mathrm{CH}_{3} \mathrm{CN}$. Due to the scattering of the nano-systems in the UVvis region of the spectrum, the decrease in the higher-energy bands $(250 \mathrm{~nm}$ to $300 \mathrm{~nm}$ ) that are characteristic of the ringclosing reaction of diarylethenes (see the spectra of $\mathbf{1 0}$ and $\mathbf{1 c}$ in Fig. 3a) cannot be observed. In either case, the appearance of the visible bands explains the change in colour of both solutions from colourless to yellow. The spectral changes stop within a minute $(30 \mathrm{~s}$ for 1o-NP in water and $60 \mathrm{~s}$ for $10 \mathrm{in}$ 
(a)
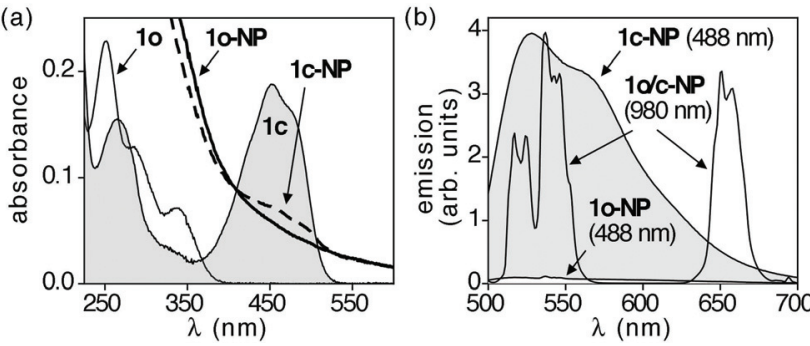

Fig. 3 (a) UV-vis absorption spectra of a $\mathrm{CH}_{3} \mathrm{CN}$ solution $\left(1 \times 10^{-5} \mathrm{M}\right)$ of chromophore 10 before (white shaded area) and after exposure to $365 \mathrm{~nm}$ light (grey shaded area), and an aqueous dispersion $\left(0.8 \times 10^{-6}\right.$ $M)^{* *}$ of the nano-assemblies (10-NP) before (solid line) and after exposure to $365 \mathrm{~nm}$ light (broken line). (b) Emission spectra for an aqueous dispersion of the nano-assemblies $\left(0.8 \times 10^{-6} \mathrm{M}\right)^{\star *}$ when excited at $488 \mathrm{~nm}$ and $980 \mathrm{~nm}$ before (10-NP) and after exposure to $365 \mathrm{~nm}$ light (1c-NP). Because the emission from the UCNPs are not affected by the states of the organic chromophores, the two spectra generated with NIR light (10/c-NP) are shown as superimpositions. The concentrations reported here are concentrations of 10 in free solution or in encapsulated nano-assemblies.

$\mathrm{CH}_{3} \mathrm{CN}$ ) at which time the chromophores have reached their photostationary states. While the amount of ring-closed isomer in the photostationary state of the nano-assmblies (1cNP) could not be directly measured, it is safe to assume the behaviour is similar to that of the free chromophores, ${ }^{8 b, d}$ which ring-close to generate 1c quantitatively as measured by ${ }^{1} \mathrm{H}$ NMR spectroscopy. Based on this assumption, the average loading in 10-NP was calculated to be 65 chromophores per nanoparticle, which is in accordance with the number of organic molecules encapsulated in the previous reported nano-assemblies synthesized by similar methods. ${ }^{8 a, b, d}$ When either coloured solution (1c-NP in water and $\mathbf{1 0}$ in $\mathbf{C H}_{3} \mathrm{CN}$ ) is exposed to visible light of wavelengths greater than $434 \mathrm{~nm}$, $\|$ it reverts back to its colourless state as the ring-open isomers are regenerated. This ring-closing/ring-opening cycle can be repeated without major changes in the spectra (Fig. S6†).

The emission behavior of the nano-assemblies is illustrated in Fig. 3b. When the aqueous dispersion of 1o-NP is excited with $980 \mathrm{~nm}$ light, it generates green emission (also shown in photographs in Fig. S4 and S5†). This emission is attributed solely to the UCNPs since neither chromophore 10 nor 1c absorbs NIR light, and exposing a $\mathrm{CH}_{3} \mathrm{CN}$ solution of 1c produces no observable fluorescence. As is typical for $\mathrm{NaYF}_{4}: \mathrm{ErYb} /$ $\mathrm{NaYF}_{4}$ nanoparticles, two sets of relatively sharp bands are observed in the region between 500 and $700 \mathrm{~nm}$ (Fig. 3b). The higher energy green emission (504-568 nm) correspond to the $\left[{ }^{2} \mathrm{H}_{11 / 2},{ }^{4} \mathrm{~S}_{3 / 2}\right] \rightarrow{ }^{4} \mathrm{I}_{15 / 2}$ transitions, while the lower energy red emission (627-684 nm) are a result of the ${ }^{4} \mathrm{~F}_{9 / 2} \rightarrow{ }^{4} \mathrm{I}_{15 / 2}$ transitions.

When light at $488 \mathrm{~nm}$ is used as excitation source, no fluorescence is observed from the aqueous dispersion of 10-NP as expected since neither component (10 or the UCNPs) absorbs at $488 \mathrm{~nm}$ (Fig. 3b). After exposure to $365 \mathrm{~nm}$ light to trigger the ring-closing reaction $(\mathbf{1 0 - N P} \rightarrow \mathbf{1 c}-\mathbf{N P})$ an intense, broad emission band between 500 and $700 \mathrm{~nm}$ is observed when the sample is excited at $488 \mathrm{~nm}$. This behavior parallels that of the free chromophore in $\mathrm{CH}_{3} \mathrm{CN}$ (Fig. S3 $\dagger$ ). The emission of 1c-NP when it is excited with $980 \mathrm{~nm}$ light was almost identical to that of 10-NP (Fig. S3†), indicating the emission from UCNPs in the nano-assemblies are not effected by the states of surrounding diarylethene molecules. The emission from the organic chromophores could be turned 'off' again by exposing the aqueous dispersion to light at wavelengths greater than $480 \mathrm{~nm}$ to regenerate the ring-open isomer (10-NP).

The use of the nano-systems as both conventional and 'turn-on' fluorescent probes was demonstrated using Caenorhabditis elegan nematodes L4 hermaphrodites, which were incubated for $3 \mathrm{~h}$ with 10-NP in $\mathrm{NaCl}$ buffer. Importantly, the nematodes were still viable after the treatment and incubation. After immobilizing the nematodes with $\mathrm{NaN}_{3}$ in $\mathrm{NaCl}$ buffer, they were washed and isolated by centrifugation, then mounted on agarose gel pads and imaged by using 2-photon fluorescence microscopy. The results are shown in the images in Fig. 4.

As anticipated, no fluorescence was observed when the animals are exposed to $488 \mathrm{~nm}$ light. However, the emission resulting from the upconverting process of NIR light by the UCNPs in 10-NP could be clearly observed in the digestive tract of the nematodes, indicating that they had ingested the nanoparticles. Exposing the animals to 365 light for $30 \mathrm{~s}$ results in clearly observable emission when excited at $488 \mathrm{~nm}$ due to the ring-closing reaction already demonstrated. Once again, the emission from the UCNPs was not effected. Importantly, the

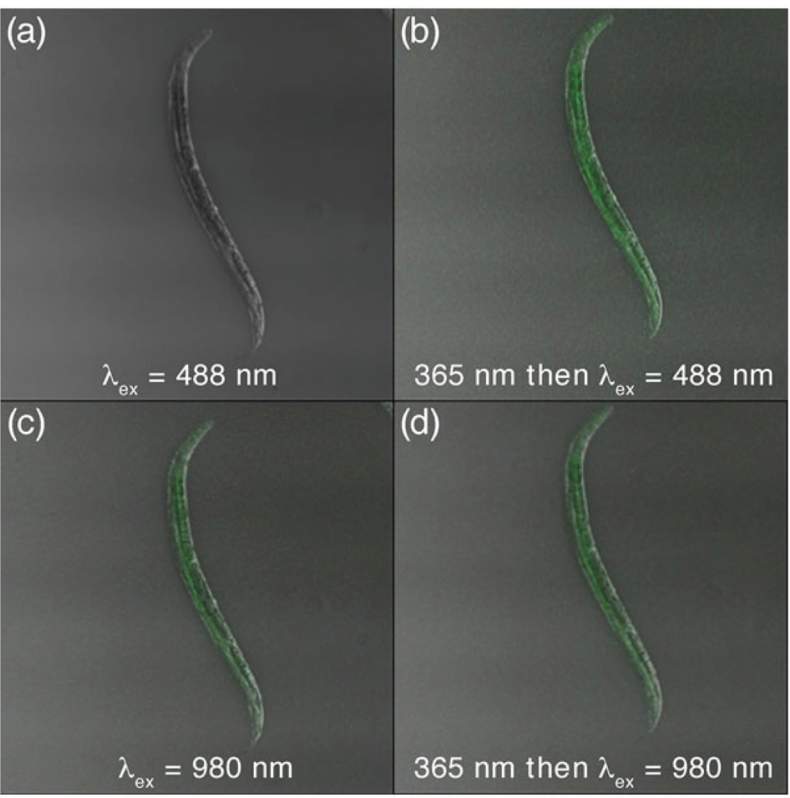

Fig. 4 Fluorescence microscopy images of a representative C. elegan when excited with $488 \mathrm{~nm}$ light (a) before and (b) after exposure to $365 \mathrm{~nm}$ light, and when excited with $980 \mathrm{~nm}$ light (c) before and (d) after exposure to $365 \mathrm{~nm}$ light. The images are the superimpositions of white light images and fluorescent images. 
entire process caused no observable damage to the nematodes, and the nematodes were still mobile before, during, and after the imaging experiments.||

\section{Conclusions}

In this article, we show how we have successfully used waterdispersible nano-assemblies containing photoresponsive diarylethene chromophores and upconverting nanoparticles as dual-functioning fluorescent probes for live organism imaging. The NIR-generated emission from the UCNPs can be used as one (static) probe as it does not change. The second emission can be modulated by toggling the photoresponsive compound between its dark (10) and bright (1c) state using UV and visible light. Systems such as this not only have the capacity to enhance the quality of images through 'turn-on' mechanism, ${ }^{2}$ they can also reduce false positive signals due to the presence of a second probe.

\section{Acknowledgements}

This research was supported by the Natural Sciences and Engineering Research Council (NSERC) of Canada and the Canada Research Chairs Program. This work made use of 4D LABS shared facilities supported by the Canada Foundation for Innovation (CFI), British Columbia Knowledge Development Fund (BCKDF) and Simon Fraser University. We thank Dr Saeid Kamal for the help with two-photon microscopy work.

\section{Notes and references}

$\$$ See ESI $\dagger$ for details.

$\S$ Standard hand-held lamps used for visualizing TLC plates were used to carry out the ring-closing and photolysis reactions at $365 \mathrm{~nm}\left(1.4 \mathrm{~mW} \mathrm{~cm} \mathrm{~cm}^{-2}\right)$. Irradiation was carried out in low-light conditions to minimize interference from ambient light.

qVisible light irradiation was carried out using the light of a $150 \mathrm{~W}$ tungsten source that was passed through a $434 \mathrm{~nm}$ cutoff filter to eliminate higher-energy light. Irradiation was carried out in low-light conditions to minimize interference from ambient light.

$\|$ The quantity of $\mathrm{NaN}_{3}$ buffer in the experiment was carefully controlled to assure paralyzing the nematodes to the level where they were immobile enough to take pictures with good resolution and focus while not killing them.
** This value is the total concentration of organic chromophores entrapped within the nano-assemblies obtained using the concentration of the ring-closed isomer (1c) at its photostationary state (as measured by UV-vis spectroscopy) divided by percentage of ring-closed isomers in this state (as measured by ${ }^{1} \mathrm{H}$ NMR spectroscopy). See ESI† for details.

1 R. Weissleder and M. J. Pittet, Nature, 2008, 452, 580.

2 H. Kobayashi and P. L. Choyke, Acc. Chem. Res., 2011, 44, 83 .

3 (a) H. Bo, M. Bates and X. Zhuang, Annu. Rev. Biochem., 2009, 78, 993; (b) M. Fernandez-Suarez and A. Y. Ting, Nat. Rev., 2008, 9, 929; (c) F. M. Raymo, Phys. Chem. Chem. Phys., 2013, 15, 14840.

4 (a) X. Chen, T. Pradhan, F. Wang, J. S. Kim and J. Yoon, Chem. Rev., 2012, 112, 1910; (b) J. Chan, S. C. Dodani and C. J. Chang, Nat. Chem., 2012, 4, 973; (c) M. E. Jun, B. Roy and K. H. Ahn, Chem. Commun., 2011, 47, 7583.

5 Y. Hori and K. Kikuchi, Curr. Opin. Chem. Biol., 2013, 17, 644.

6 K. Uno, H. Niikura, M. Morimoto, Y. Ishibashi, H. Miyasaka and M. Irie, J. Am. Chem. Soc., 2011, 133, 13558 .

7 (a) M. Irie, T. Fukaminato, K. Matsuda and S. Kobatake, Chem. Rev., 2014, 114, 12174; (b) B. L. Feringa, Molecular Switches, Wiley-VCH, Weinheim, 2010; (c) L. Ubaghs, D. Sud and N. R. Branda, Handbook in Thiophene-Based Materials: Applications in Organic Electronics and Photonics, ed. I. D. Perepichka and D. Perepichka, John Wiley \& Sons, Chichester, 2009, vol. 2; (d) H. Tian and S. Yang, Chem. Soc. Rev., 2004, 33, 85.

8 (a) T. Wu, S. Kaur and N. R. Branda, Org. Biomol. Chem., 2015, 13, 2017; (b) T. Wu, D. Wilson and N. R. Branda, Chem. Mater., 2014, 26, 4313; (c) T. Wu, M. Barker, C.-J. Carling, K. M. Arafeh, J.-C. Boyer and N. R. Branda, Angew. Chem., Int. Ed., 2013, 52, 11106; (d) T. Wu, J.-C. Boyer, M. Barker, D. Wilson and N. R. Branda, Chem. Mater., 2013, 25, 2495.

9 (a) M. Nyk, R. Kumar, T. Y. Ohulchanskyy, E. J. Bergey and P. N. Prasad, Nano Lett., 2008, 8, 3834; (b) M. Haase and H. Schäfer, Angew. Chem., Int. Ed., 2011, 50, 5808; (c) S. Gai, C. Li, P. Yang and J. Lin, Chem. Rev., 2014, 114, 2343; (d) Y. Park, K. T. Lee, Y. D. Suh and T. Hyeon, Chem. Soc. Rev., 2015, 44, 1302.

10 J.-H. Lee and J. Cheon, Anal. Sci. Technol., 2011, 2, A71. 